\title{
CFD BASED EXPLOSION RISK ANALYSIS USING MULTI-DIMENSIONAL FREQUENCY DISTRIBUTION OF FLAMMABLE GAS CLOUDS
}

\author{
YANLIN JIN ${ }^{1} \&$ BEOM-SEON JANG ${ }^{2}$ \\ ${ }^{1}$ Department of Naval Architecture and Ocean Engineering, Seoul National University, South Korea \\ ${ }^{2}$ Research Institute of Marine Systems Engineering, Seoul National University, South Korea
}

\begin{abstract}
Explosion risk analysis (ERA), one of the dedicated safety studies in offshore risk assessment, aims at evaluating the design accidental loads (DALs) for offshore topside structures and facilities. This study is an effort to propose a new type of flammable gas cloud frequency distribution appeared ERAs for offshore topside process. Flammable gas cloud distribution is a kind of intermediate result which is used for investigating a number of appropriate explosion scenarios by incorporating the results of frequency analysis, gas dispersion and ignition probability modelling. It is well known that the consequences of explosion accidents may vary strongly depending on variables such as the locations of ignition sources, gas cloud positions, sizes, and shapes, etc. This study is devoted to presenting an appropriate way to quantify the gas cloud position by using the results of dispersion simulations and reflect them into the proposed distribution to break through what were only addressed qualitatively by engineering judgement before. Using the proposed distribution, frequency of explosions is distributed over the gas cloud size and the position, and one can therefore expect a number of more realistic explosion scenarios which may improve the accuracy of DALs to a certain extent.

Keywords: explosion, risk, ERA, DAL, gas cloud distribution.
\end{abstract}

\section{INTRODUCTION}

Many offshore platforms are normally exposed to the flammable oil and gas circumstance and hence explosions caused by hydrocarbon leakage are big threats to the offshore platforms. Since the problems of using the worst-case studies and simplified gas dispersion model are addressed by many full-scaled explosion experiments, CFD based probabilistic approaches have become more and more practical in gas explosion risk analyses (ERAs). However, probabilistic approaches have a challenge with an infinite number of explosion scenarios in common, and as an alternative, in current methodologies, a concept of gas cloud distribution [2]-[4] is adopted to investigate a number of representative explosion scenarios. The distribution can be derived by a finite number of leak scenarios, using the results of frequency analysis, CFD gas dispersion simulations and ignition probability modelling.

The gas cloud distribution is widely accepted by oil and gas industry, because it is a practical way to handle the infinite number of actual explosion scenarios efficiently and economically. Nevertheless, there is some difference between the actual explosion scenarios and the ones investigated by the gas cloud distribution. The difference mainly resides in some important variables which might be ignored when the explosion scenarios are investigated through the distribution. In general, explosion consequences can vary an order of magnitude or even more for the same gas cloud sizes depending on the locations of ignition sources, gas cloud positions and shapes of gas cloud, etc. In most of current methods, the gas cloud distribution is a two-dimensional frequency distribution, which illustrates that explosion frequency is only dependent on the gas cloud sizes. With this distribution, however, there is often a number of size-position mismatched gas clouds in investigated explosion scenarios, and this may lead to overestimated or underestimated explosion loads. 
To obtain a better solution, in the current study, a multi-dimensional gas cloud frequency distribution is proposed that includes both the position and size of gas clouds to distribute the explosion frequency. When explosion scenarios are investigated by the proposed distribution, the information of the gas cloud position is taken into account automatically, and therefore one can expect that the investigated explosion scenarios no longer comprise the size-position mismatched gas clouds. Details on how to get the proposed distribution is illustrated in this study, and case studies are demonstrated to compare the results of ERA, i.e. exceedance curves evaluated by using both the existing and proposed distributions, respectively.

\section{EXISTING APPROACH}

There are a lot of variables associated with the explosion scenarios occurring in offshore topside process areas. Typically, these variables can be operating condition, leak hole size, leak direction, wind speed, wind direction, ignition location, mass of inventory, etc. Upon gas leakages, most of the above-mentioned variables appear randomly and thereby it is hard to predict them correctly. To solve this, probabilistic approaches [2]-[4] become more and more popular in ERA methodologies. Compared to the worst-case studies, probabilistic approaches give more acceptable results, which is less conservative than the worst-case studies [4]. Following Fig. 1 shows an overall procedure of probabilistic explosion risk analysis used in this study, which is figured out by previous researches [2]-[4] and some standard guidelines [5].

Implication of Fig. 1 is that the ERAs require a complicated process which should cover the entire chain of events starting from a leak, via dispersion, ignition and explosion itself. The whole process can be divided into two parts based on the point of gas cloud distribution. The first part is a sub-process for obtaining the gas cloud distribution which is derived by combining the results of the leak frequency analysis, ignition probability modelling and dispersion simulation. Fig. 2 represents an example of the gas cloud distribution determined by existing approaches, which is comprised only by the gas cloud size and the explosion frequency.

The other sub-process is devoted to investigate the explosion scenarios and perform the explosion simulations to evaluate explosion loads. The reason for taking gas cloud distribution as a base point to separate the whole ERA process is that the gas cloud distribution as a turning point, contributes a lot to reducing the total number of the explosion scenarios to a manageable level.

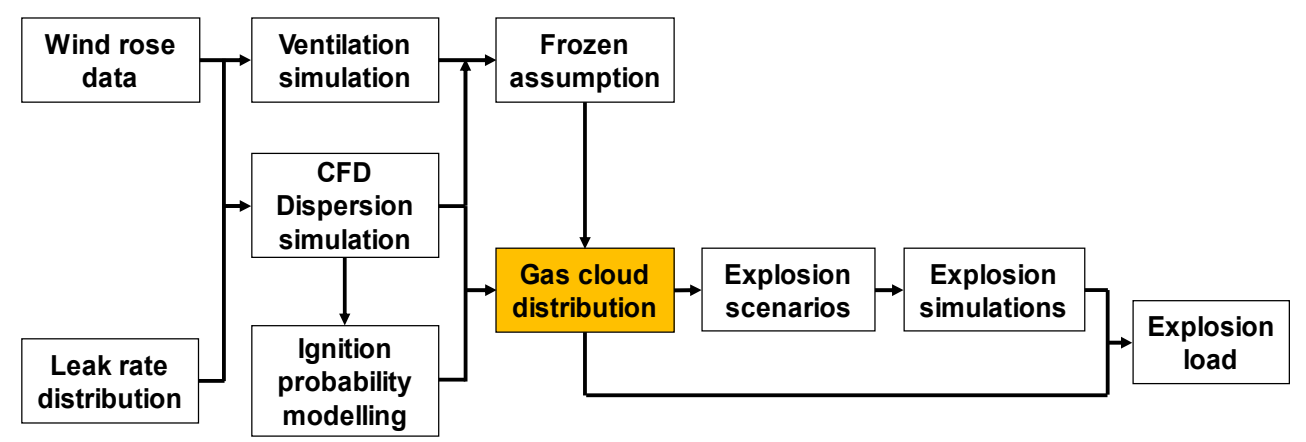

Figure 1: Procedure of probabilistic explosion risk analysis. 


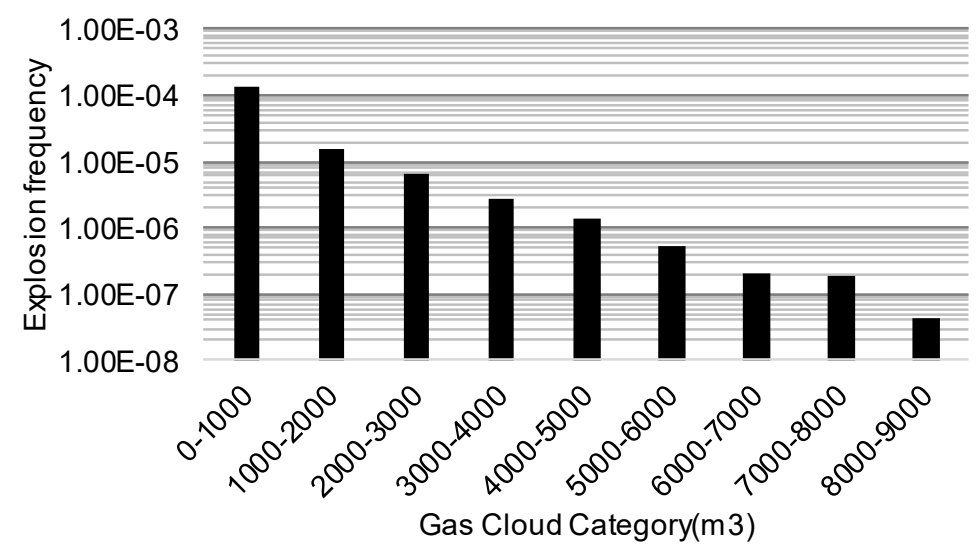

Figure 2: Existing gas cloud distribution.

It is fair to say that the gas dispersion simulation is at least as challenging as the explosion simulation because both of them require numerous input scenarios. For leak scenarios, there are also some practical approaches to reduce its total number, and the frozen assumption presented in Fig. 1 can be one of the representatives. However, the current study only focuses on the later sub-process of the ERAs, which covers how to evaluate the gas cloud distribution more elaborately from the onset of a finite number of given dispersion simulations in order to evaluate the explosion loads accurately.

\subsection{Existing gas cloud distribution}

The total number of leak scenarios can be infinite, but in most cases it can be under control by some probabilistic approaches [2]. However, different from gas dispersion, the explosion itself is an instantaneous phenomenon, which means that it is likely to take place in every single moment during the gas dispersion, depending on dispersed gas plumes and ignition source distributions. Therefore, in theory, as long as the gas is dispersed, the total number of explosion scenarios can be infinite no matter how many gas leak scenarios are considered. Due to such a feature, it is in fact impossible to accomplish the explosion simulations directly by using the results of dispersion simulations. In order to reduce the number of explosion scenarios, and meanwhile reflecting all possible gas cloud formations properly, the concept of the gas cloud distribution is come up with in probabilistic ERAs. In simple terms, the gas cloud distribution is a discrete frequency distribution for gas cloud size, which provides the sum of the frequency of the gas cloud sizes located in a certain range. Using this frequency distribution, the numerous cases of gas cloud size can be under control probabilistically. In general, the gas cloud distribution can be readily obtained by grouping the original numerous gas clouds into a certain number of gas cloud size category, and summing all of the associated individual gas cloud frequencies for each category.

\subsection{Explosion scenarios}

The purpose of the explosion simulation is to calculate the explosion loads that can occur on various structures or equipment locating in the target topside process. The loads can be overpressure, impulse or some other types of dominating force that might damage the 
structures or equipment. Normally, these forces are presented together with the explosion frequency as exceedance curves to demonstrate the design accidental loads (DALs). In probabilistic ERAs, explosion simulations are performed with target process area for varying gas cloud sizes, gas cloud positions and ignition locations. The gas clouds can vary from the lowest to the highest cloud size category and locating through the full target process area. Ignitions at both the central and edge locations of each cloud are considered. By present, there is not any specific methodologies for choosing the right cloud positions or ignition locations, but in most cases, they are more likely to be determined in a qualitative manner by engineering judgements which is complied with standardized guidelines and recommended practices. The authoritative NORSOK standard [5], recommends that the frequency distribution of gas cloud locations shall take into account the location of leak sources and ventilation conditions, e.g. wind rose, etc., but neither does it clarify any specific methodologies. Except for some specific cases, in each gas cloud category, the gas cloud position is normally determined by a certain number of cases that are evenly distributed throughout the full target process area. The number of positions determined will be different depending on the cases, but the sum of their frequency should be kept consistent with the frequency of the corresponding category.

\subsection{Problems of accuracy}

In order to calculate fairly valid explosion loads, going back to the beginning, the leak scenarios should be determined in a proper manner so as to make the gas cloud distribution be representative enough for explosion simulations. However, no matter how properly the leak scenarios are determined, there is still a difference in gas cloud data between the dispersion simulation results and the investigated explosion scenarios. Typical example of this can be the size-position mismatched gas clouds shown in Fig. 3.

In this example, 5 cases of gas cloud position are considered for each gas cloud category, and they are evenly distributed throughout the process area. According to the dispersion simulations, if the shaded region is regarded as the highest category appeared region, the $5^{\text {th }}$ gas cloud in the figure will probably lead to overestimation of loads for the surrounding targets or conversely underestimation of loads for the targets locating far from the $5^{\text {th }}$ cloud. This is because explosion with the $5^{\text {th }}$ gas cloud will produce a higher overpressure than it should experience for the surrounding targets; whereas reduce the portion of the frequency of the rest gas clouds, which might consequently lower the exceedance curve for the targets far from the $5^{\text {th }}$ cloud. More details about the problem of size-position mismatched gas clouds are described in Section 4.

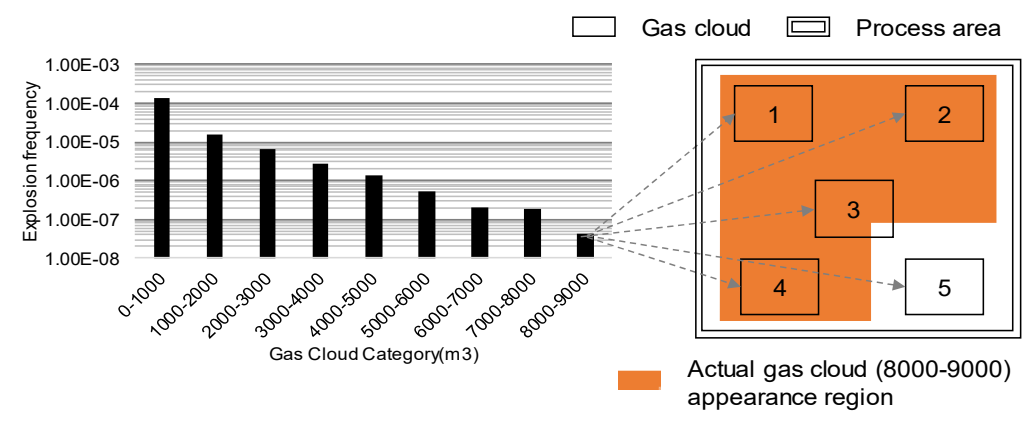

Figure 3: Determination of gas cloud position with existing gas cloud distribution. 


\section{CURRENT APPROACH}

To fix the improper consideration of the gas cloud position in existing approaches, the current study proposes a new type of gas cloud distribution, i.e. multi-dimensional frequency distribution (MDF), which includes both the data of cloud size and position so as to avoid the problematic clouds.

\subsection{Calculation of gas cloud position}

The main idea of proposed MDF is to separate the frequency not only by the size of the gas cloud, but also by the associated gas cloud position. In order to do so, the gas cloud position should be quantified first. In the current study, the gas cloud position is defined as the volumetric centre of equivalent stoichiometric gas cloud [6], [7] and by using the results of CFD gas dispersion simulation results, the centre of the gas cloud is calculated. For convenience, an in-house code is developed to accomplish the calculation. The code is interfaced with a commercial CFD tool, i.e. FLACs [7] and computation is taken place at every single time step of CFD simulation to export a complete time trajectory of the centre. Following Fig. 4 shows an example of gas cloud centre positions at 4 different snapshots. The contour shows the distribution of gas concentration expressed by a normalized flammable range with an equivalence ratio (EQNFL) [7]. EQNFL=1 stands for the upper flammable limit, whereas EQNFL=0 stands for the lower flammable limit. The calculation only considers the gas concentration belonged to flammable range.

\subsection{Multi-dimensional frequency distribution}

As long as the position of every single gas cloud is available to be quantified, the proposed MFD is readily attainable by using a similar sequence applied for obtaining the existing gas cloud distribution. The detailed procedure is described by following several steps:

- Step 1: Monitoring the time evolution of non-homogeneous gas cloud size in all prescribed leak scenarios with a certain time interval.

- Step 2: Calculate the time history of ignition probability.

- Step 3: Calculate equivalent gas cloud [6], [7] size and associated centre for each monitored gas cloud.

- Step 4 Calculate explosion frequency for each gas cloud.

- Step 5: Specify the number and range of category for gas cloud size.

- Step 6: Divide the entire domain of target process area into $\mathrm{n} \times \mathrm{m} \times 1$ subspaces. ( $\mathrm{n}, \mathrm{m}, \mathrm{l}$ stands for the number of intervals used for dividing the domain in $\mathrm{x}, \mathrm{y}, \mathrm{z}$ direction, respectively.)

- Step 7: Distribute the full set (i.e. size, position) of the gas cloud by using the categories defined in Step 5 and 6, and evaluate the sum of frequency in each combination.

In Step 1, a time interval should be specified for monitoring the gas cloud. The smaller value chosen, the more realistic gas cloud distribution can be attained. Practically, consistent with another time interval used in the calculation of the ignition probability, the value can be practically chosen as 1s. In the current study, ignition probability is calculated by using the time dependent ignition probability model (TDIIM) [8], [9], which evaluates the probability by considering the gas cloud propagation and all potential ignition sources together. Some examples of the proposed MFD is presented in Section 4. 

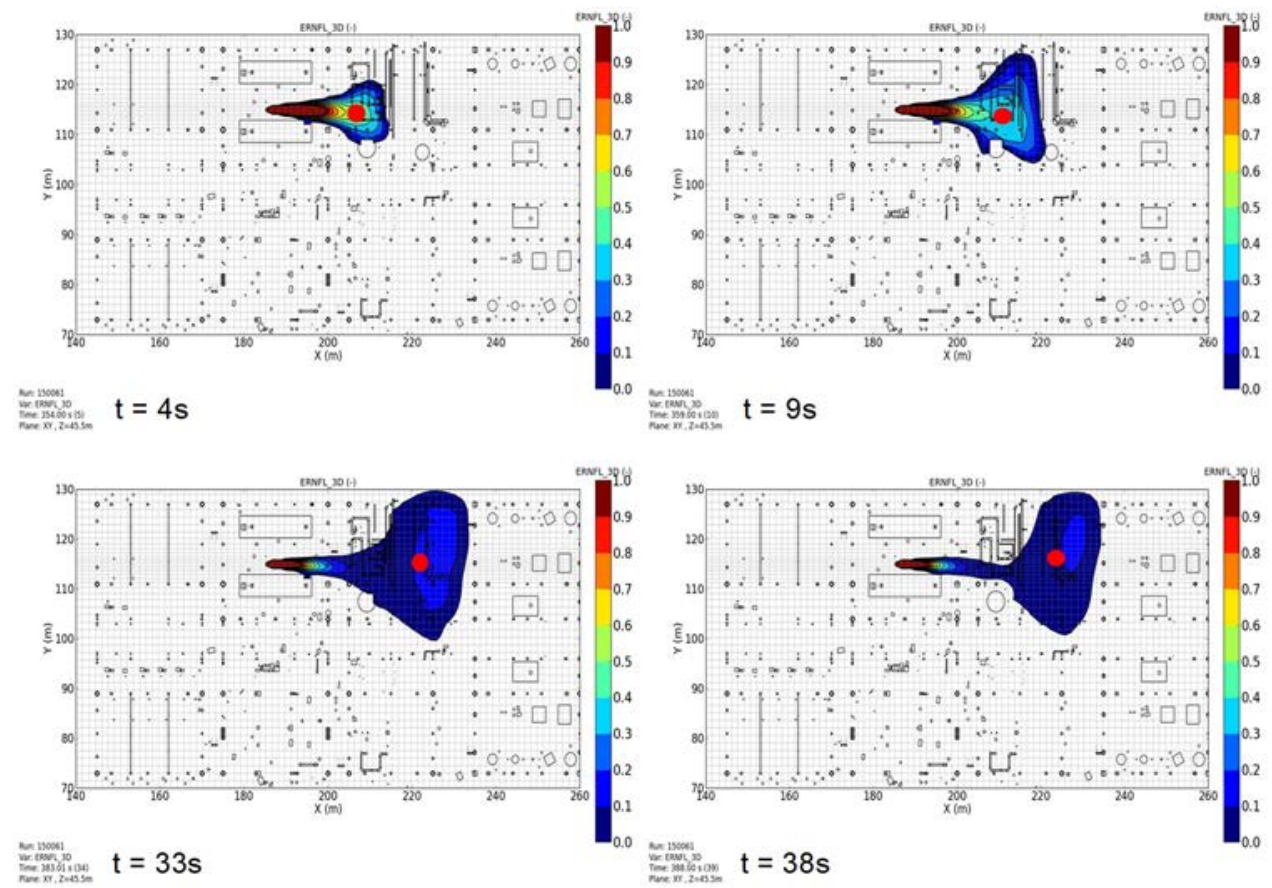

Figure 4: Centre of gas cloud position.

\section{CASE STUDY}

In this section, some cases are studied to compare the results of exceedance curve determined by both the existing and the proposed gas cloud distribution, respectively. By using the two kinds of distribution, 3 sets of explosion scenarios are investigated, and the results of explosion simulation are used to evaluate the exceedance curves in several prescribed locations.

\subsection{Evaluation of gas cloud distribution}

In order to get the gas cloud distributions, a small number of leak scenarios are considered for a sub-part of the target FPSO topside module shown in Fig. 5. There will be tremendous leak scenarios in real situations; however, for simplicity only 2 typical process segments with 2 leak positions, 5 leak directions and 5 leak holes are reflected in the investigated leak scenarios. Wind condition is also given by only 1 case of direction and speed. The applied initial leak rates by different leak holes are summarized in Table 1. Dispersion simulation is carried out by using the time dependent leak rate [10] and lasts until the end time of each leak profile. In this example, the total number of leak scenario is 50 , whereas the total number of appeared gas cloud reaches up to 20475, which means that the sum of leak durations for 50 leak scenarios is 20475 s in total. Fig. 2 is the result of gas cloud distribution by using existing approach. The total frequency is distributed by 9 different gas cloud size categories. It is obvious to observe that the portion of the frequency is decreased exponentially from the lowest to the highest category. 


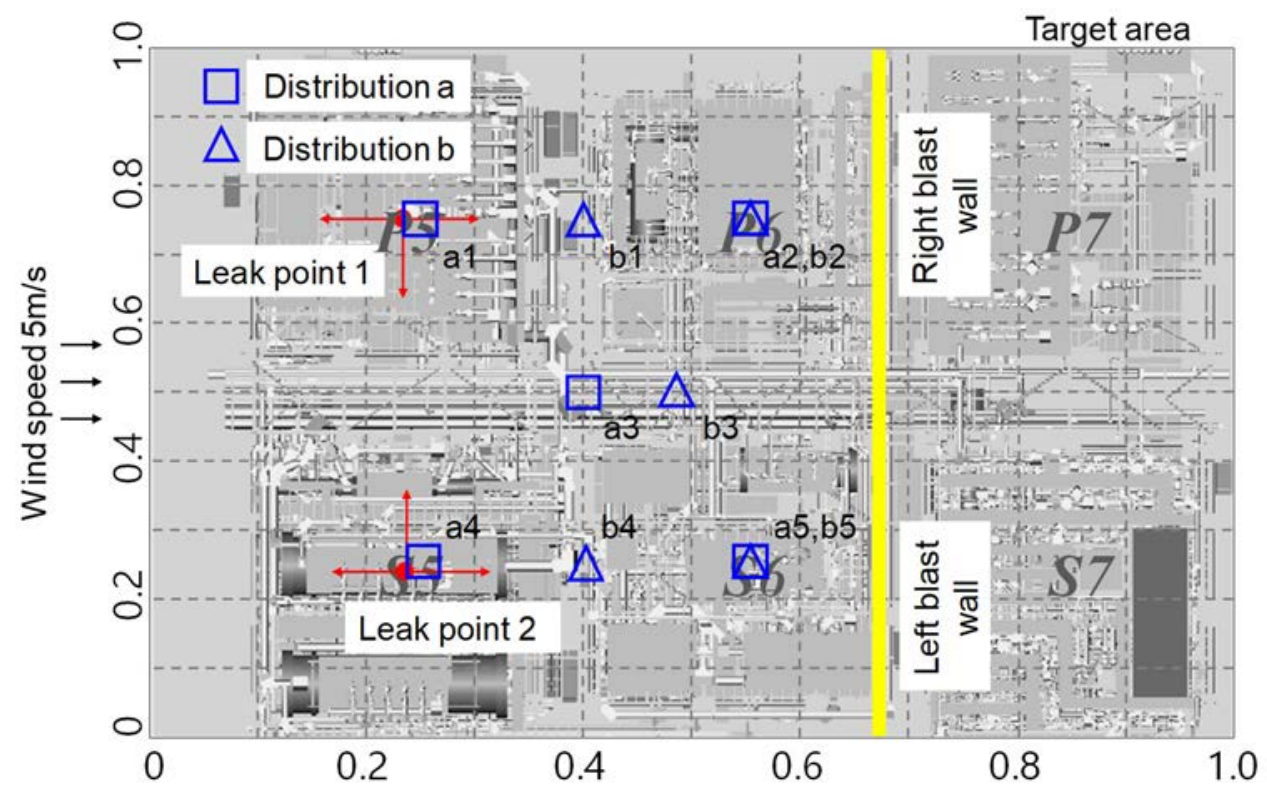

Figure 5: Target FPSO topside process area.

Table 1: Leak rate category.

\begin{tabular}{|l|l|l|}
\hline Category & P5 & S5 \\
\hline $2-4 \mathrm{~kg} / \mathrm{s}$ & $3.39 \mathrm{~kg} / \mathrm{s}$ & $3.55 \mathrm{~kg} / \mathrm{s}$ \\
\hline $4-8 \mathrm{~kg} / \mathrm{s}$ & $6.44 \mathrm{~kg} / \mathrm{s}$ & $6.31 \mathrm{~kg} / \mathrm{s}$ \\
\hline $8-16 \mathrm{~kg} / \mathrm{s}$ & $13.57 \mathrm{~kg} / \mathrm{s}$ & $14.20 \mathrm{~kg} / \mathrm{s}$ \\
\hline $16-32 \mathrm{~kg} / \mathrm{s}$ & $30.53 \mathrm{~kg} / \mathrm{s}$ & $31.93 \mathrm{~kg} / \mathrm{s}$ \\
\hline $32-64 \mathrm{~kg} / \mathrm{s}$ & $54.27 \mathrm{~kg} / \mathrm{s}$ & $56.76 \mathrm{~kg} / \mathrm{s}$ \\
\hline
\end{tabular}

In the proposed gas cloud distribution, the position of gas cloud is expressed by 3 independent variables, i.e. $\mathrm{x}, \mathrm{y}$ and $\mathrm{z}$, respectively. This means that the proposed distribution is a 5-dimensional distribution; therefore, it is practically impossible to plot this distribution completely. However, purely for visual presentation, a simplified version of the proposed distribution is plotted in Fig. 6. In these figures, it is assumed that the gas cloud moves only along the $x-y$ plane, and the portion of frequency for gas cloud size category is distributed on normalized x-y domain presented in Fig. 5. Obviously, in both of the 2 plots, it is clear to see that there are not any gas clouds locating beyond 0.6 in $\mathrm{x}$ direction, and this can be explained by the blast wall locating between 0.6 and 0.8 , which prevents the gas clouds from moving forward. Fig. 6(a) and Fig. 6(b) stands for the first and last category presented in Fig. 2, respectively. The same type of plot is also available for the rest gas cloud categories. 


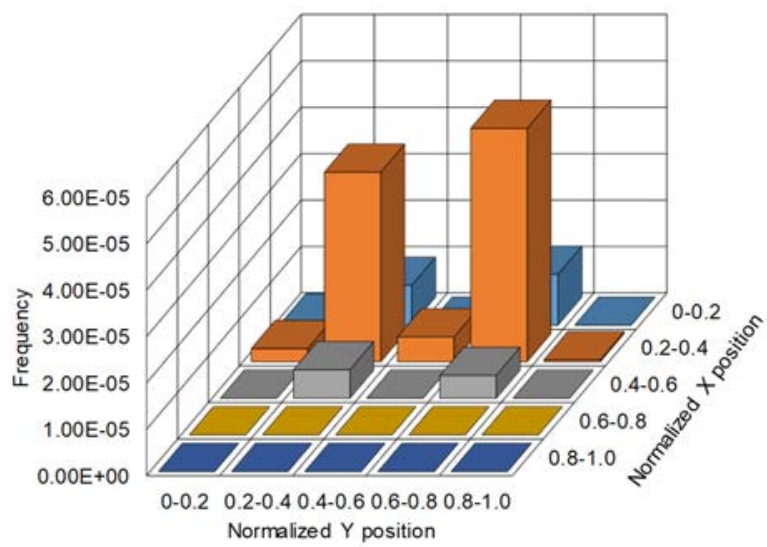

(a)

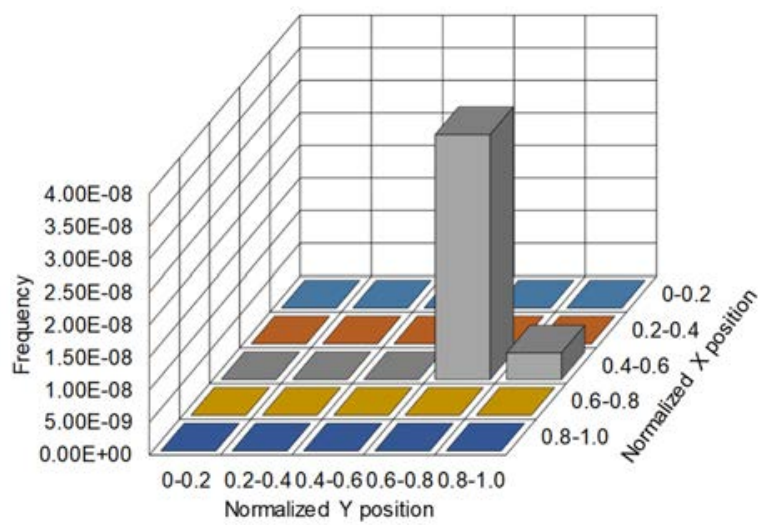

(b)

Figure 6: Target FPSO topside process area.

\subsection{Explosion scenarios}

In this case study, 3 sets of the explosion scenarios are investigated by using the gas cloud distributions evaluated in Section 4.2, and among them, 2 alternative distributions of gas cloud position are considered with the existing distribution. The details of these distributions are marked in Fig. 5, both of which are located throughout the target area by including 5 different positions $1-5$, respectively. Compared to distribution a, distribution $b$ is shifted to the blast wall, which illustrates that distribution $b$ adopts more of the wind effect. By locating the 9 categories of gas cloud size in each position, meanwhile, considering 1 ignition point, i.e. central ignition, the total number of investigated explosion scenarios with existing gas cloud distribution is 45 .

In contrast, by using the proposed MDF, the total number of investigated explosion scenarios will be 1125 , where 5 subspaces are considered in each spatial coordinate, respectively. However, among these scenarios, most of which have zero frequency and hence, the actual number of valid scenarios will be reduced to 105 . 


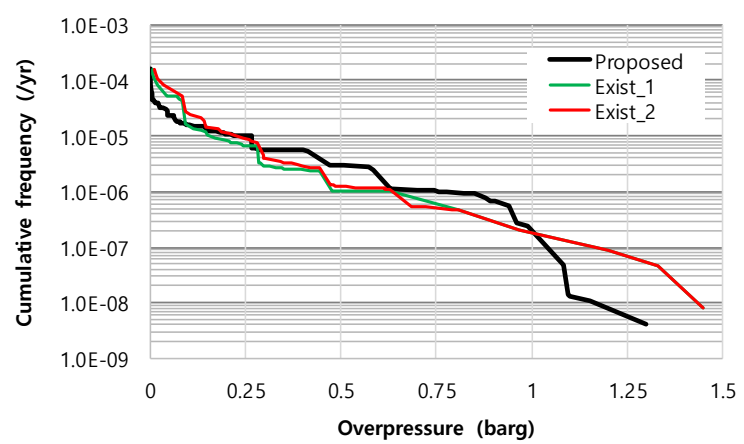

(a)

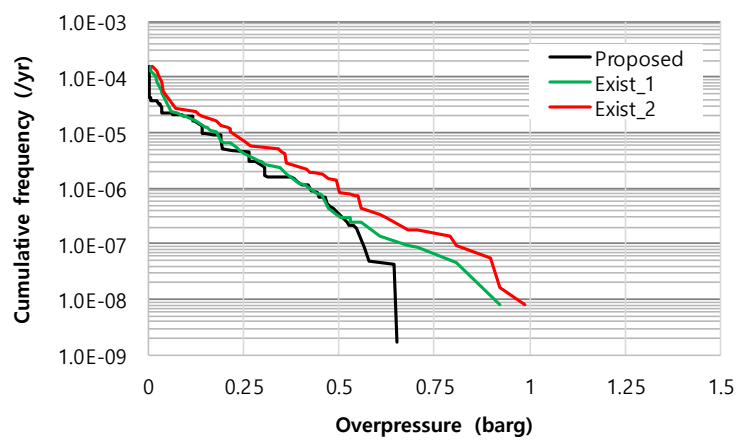

(b)

Figure 7: Overpressure exceedance curves.

\subsection{Evaluation of exceedance curve}

3 cases of the overpressure exceedance curve are evaluated by using the results of explosion simulation. The overpressure on the blast wall is monitored by two groups of evenly distributed monitoring points locating in front of the left and right part of blast wall. The exceedance curves are evaluated based on the maximum values of overpressure experienced by each sub-part of the blast wall. These maximum values are extracted from the monitoring data of every investigated explosion scenario. Fig. 6 presents the final evaluated exceedance curves; (a) shows the left part of the blast wall's exceedance curve while (b) shows the right part of the blast wall's exceedance curve. As illustrated by the simplified version of MDF shown in Fig. 6, the high level of gas cloud size category is not appeared in position a5, b5. However, the 2 alternative distributions of gas cloud position considered in existing approaches show that the high level of category is included in position a5 and b5, which indicates the typical problem of size-position mismatched gas clouds described in Section 2.3. As discussed in Section 2.3, the existing approach overestimates the exceedance curve of the left part of the blast wall; whereas, underestimates the right part of the blast wall as which are located far from the position a5, b5.

\section{CONCLUSION}

Explosion risk analysis is challenging with numerous input scenarios, and to solve that, a concept of gas cloud distribution is adopted to indirectly reflect the results of dispersion 
simulation for investigating the explosion scenarios. However, existing gas cloud distribution leads to a problem of having cloud size-position mismatched gas clouds, which might cause to overestimate or underestimate the explosion loads. As a solution, the current study proposed a new type of gas cloud distribution, i.e. MDF. By adding the position of the gas cloud as another variable in the gas cloud distribution, the investigated explosion scenarios are free from the problem. The results of case studies conclude that explosions with the problematic gas clouds in the existing distribution produce a higher overpressure than it should experience for the surrounding targets, whereas reduce the portion of the frequency of the rest gas clouds, which might consequently lower the exceedance curve for the targets far from them. With the case studies, it is proved that the proposed MDF can produce more accurate explosion loads than the existing gas cloud distribution. As a future work, a variety of applications to different models need to be carried out to further verify the accuracy and efficiency of the proposed approach.

\section{ACKNOWLEDGEMENTS}

This research was respectfully supported by Engineering Development Research Centre (EDRC) (No. N0000990), and the project "Development of integrated interactive model for subsea and topside systems to evaluate the process design of offshore platform" (No. 10060099) funded by the Ministry of Trade, Industry and Energy (MOTIE).

\section{REFERENCES}

[1] Jin, Y.L. \& Jang, B.S., Probabilistic fire risk analysis and structural safety assessment of FPSO topside module. Ocean Eng, 126(7), pp. 29-46, 2015.

[2] Hansen, O.R., Talberg, O. \& Bakke, J.R., CFD-based methodology for quantitative gas explosion risk assessment in congested process areas. International Conference and Workshop on Modelling the Consequences of Accidental Releases of Hazardous Materials, San Francisco, USA, pp. 457-477, 1999.

[3] Hoorelbeke, P., Bakke, J.R., Izatt, C., Renoult, J. \& Brewerton, R.W., Vapor cloud explosion analysis of onshore petrochemical facilities. 7th Professional Development Conference and Exhibition, Kingdom of Bahrain, pp. 248-261, 2006.

[4] Davis, S.G. et al., Benefits of risk-based design through probabilistic consequence modeling. 7th Global Congress on Process Safety, Chicago, USA, 2011.

[5] NORSOK Z-013, Risk and emergency preparedness analysis. NORSOK Standard, Oslo, Norway, 2001.

[6] Hansen, O.R., Gavelli, F., Davis, S.G. \& Middha, P., Equivalent cloud methods used for explosion risk and consequence studies. J. Loss Prev. Process Ind, 26, pp. 511527, 2013.

[7] FLACS, FLACS User's Manual. Revision 10.4. GexCon A.S., Bergen, Norway, 2015.

[8] Jin, Y.L. \& Jang, B.S., Study on ignition probability model for probabilistic explosion risk analysis in congested offshore process area. Proceedings of the International Conference on Collision and Grounding of Ships and Offshore Structures, Ulsan, South Korea, 2016.

[9] Det Norske, Ignition Modelling Time Dependent Ignition Probability Model. Report No. 96-3629, 1998.

[10] Gupta, S. \& Chan, S., A CFD based explosion risk analysis methodology using time varying release rates in dispersion simulations. J. Loss Prev. Process Ind, 39(7), pp. 59-67, 2016. 